\title{
Physiochemical and Descriptive Sensory Analysis of Arkansas Muscadine Grapes
}

\author{
Molly Felts and Renee T. Threlfall ${ }^{1}$ \\ Institute of Food Science and Engineering and Department of Food Science, \\ University of Arkansas, 2650 North Young Avenue, Fayetteville, AR 72704
}

John R. Clark and Margaret L. Worthington

Department of Horticulture, University of Arkansas, 316 Plant Science, Fayetteville, AR 72701

Additional index words. Vitis rotundifolia, composition, marketability, ripeness

\begin{abstract}
Understanding how consumer perception is related to physiochemical attributes assists in the identification of harvest and marketability parameters for muscadine grapes (Vitis rotundifolia Michx.). Three muscadine cultivars (Ison, Nesbitt, and Summit) and three advanced breeding selections (AM-9, AM-74, and AM-83) were harvested from vines at the University of Arkansas System Division of Agriculture Fruit Research Station in Clarksville, AR. The physiochemical (physical and composition) and sensory attributes (descriptive) of the genotypes were evaluated at harvest. Significant differences between genotypes were observed for berry weight $(9.25-14.38 \mathrm{~g})$, soluble solids $(12.73 \%$ to $15.40 \%)$, pH $(2.88-3.33)$, titratable acidity $(0.54 \%$ to $1.01 \%)$, soluble solids/ titratable acidity ratio (13.12-28.49), skin firmness $[0.85-1.48$ Newtons/millimeters $\left.\left(\mathrm{N} \cdot \mathrm{mm}^{-1}\right)\right]$, and flesh firmness $(0.89-2.14 \mathrm{~N})$. Total sugars $(6.17-9.75 \mathrm{~g} / 100 \mathrm{~g})$ and total organic acid $(0.50-0.84 \mathrm{~g} / 100 \mathrm{~g})$ levels were not significantly different for these genotypes. A trained descriptive sensory panel $(n=8)$ evaluated the fruit attributes for aroma $(n=$ 9), external appearance $(n=7)$, internal appearance $(n=3)$, basic tastes $(n=3)$, aromatics $(n=10)$, feeling factors $(n=2)$, and texture $(n=7)$. The descriptive sensory panel detected differences among genotypes for external appearance, internal appearance, and basic taste attributes, more specifically with desirable attributes rather than unfavorable. However, the panelists found no differences among genotypes for texture attributes. Of the physiochemical attributes, total sugars had the most significant correlations with the descriptive sensory attributes, followed by soluble solids/titratable acidity ratio. Total sugars were correlated to 12 attributes (three aromas, two exterior appearances, two basic tastes, four aromatics, and one feeling factor) and soluble solids/titratable acidity was correlated to five attributes (one aroma, one basic taste, two aromatics, and one feeling factor). A lexicon of terms for descriptive sensory attributes for fresh-market muscadine grapes was established. This lexicon can be used for other research and breeding efforts, as well as establishing the relationship between the physiochemical and descriptive sensory attributes.
\end{abstract}

Because of the high humidity and incidence of disease, grapes grown for commercial production in the southern United States need to be disease tolerant. The muscadine grape ( $V$. rotundifolia Michx.) is native to the southern United States. Currently, muscadines are grown commercially in Alabama, Arkansas, Florida, Georgia, Louisiana, Mississippi, North Carolina, Oklahoma, Tennessee, Texas, South Carolina, and Virginia, and are resistant to a variety of diseases and pests (Bouquet, 1981; Olien, 1990; Ren and Lu, 2002). Muscadine production can be very profitable for southern growers, and in a 2006 profitability study, 12 U.S. southern states grew $\approx 2025$ ha of

Received for publication 14 June 2018. Accepted for publication 7 Sept. 2018.

This research was funded by a grant from The Southern Region Small Fruit Consortium.

${ }^{1}$ Corresponding author. E-mail: rthrelf@uark.edu. muscadine grapes, of which $90 \%$ were 'Carlos', a bronze-processing cultivar (Carpio et al., 2008; Cline and Fisk, 2006).

Consumer sensory evaluations on muscadines indicated that consumers liked the flavor of the grape but disliked the seeds and tough skin (Degner and Mathis, 1980). In a consumer study by Brown et al. (2016), thinner skins and higher juice $\mathrm{pH}$ were associated with greater overall liking of muscadine grapes. Consumer acceptability of muscadines can be quantified with soluble solids analysis, texture analysis, and sensory analysis (Brown et al., 2016). However, most of the studies have focused on juice rather than the whole muscadine berry (Flora, 1979; Meullenet et al., 2008; Trappey et al., 2007). In addition, limited studies have been carried out on descriptive sensory analysis of whole, fresh-market muscadine berries. Descriptive sensory analysis quantitatively describes fruit attributes, such as basic tastes, aroma, and texture, using trained panelists (Contador et al., 2017). Utilization of this method has the potential to describe how an attribute is perceived by the consumer. Descriptive sensory analysis provides valuable information for fruit breeders on fruit attributes to identify potential improvements.

The University of Arkansas's Fruit Breeding Program began breeding muscadines in 2005 with a focus on large fruit size, crisp texture, edible skin, self-fruitful flowers, seedlessness, and improved postharvest storability (Barchenger, 2015a). Increasing the consumer liking of muscadine grapes, and products produced from these grapes, is an important consideration in muscadine breeding. Newer cultivars of fresh-market muscadines with improved consumer quality attributes have the potential to expand the grape market in the United States.

Understanding the physiochemical and sensory attributes of Arkansas-grown muscadine genotypes (cultivars and advanced selections) is important to demonstrate fresh-market potential. The purpose of this study was to evaluate the physiochemical attributes and descriptive sensory attributes of fresh-market muscadine grapes at harvest.

\section{Materials and Methods}

\section{Plants and culture}

Six muscadine genotypes (AM-9, AM-74, AM-83, 'Nesbitt', 'Ison', and 'Summit') were harvested from vines grown at the University of Arkansas Fruit Research Station, Clarksville, AR [west-central Arkansas, lat. $35^{\circ} 31^{\prime} 58^{\prime \prime} \mathrm{N}$ and long. $93^{\circ} 24^{\prime} 12^{\prime \prime} \mathrm{W}$; U.S. Department of Agriculture hardiness zone $7 \mathrm{a}$; soil type: Linker fine sandy loam (Typic Hapludult)]. Vines were spaced $6.1 \mathrm{~m}$ apart and rows were spaced $3.0 \mathrm{~m}$ apart. AM74 and 'Summit' were bronze-skinned genotypes, and AM-9, AM-83, 'Ison', and 'Nesbitt' were black-skinned genotypes. Vines in the study varied in age from 5 to 25 years but were fully mature and were uniform in cropping and plant health. The vines were trained to a bilateral, high-cordon/curtain training system and pruned retaining three to four node spurs. Spur density was $\approx 12 \mathrm{~cm}$ between spurs. Weeds were controlled by applications of preemergent and postemergent herbicides applied annually. Vines were fertilized annually in March or April with nitrogen or complete fertilizers. Fungicides were applied similar to a commercial requirement to control various rots. The last application of any fungicide was usually carried out near the end of June to early July. On average, five fungicide sprays and two insecticide sprays were applied to the grapes.

\section{Harvest}

The muscadine genotypes for this study ripen about the same time and were selected so that physiochemical and descriptive sensory analysis of the six genotypes could be performed on one date. Muscadine grapes ripen asynchronously, but abscise easily from the stem when ripe. The six genotypes were harvested at optimal commercial ripeness 
(removed easily from the stem, shiny, and fully colored) for fresh-market muscadine grapes. The fruit was free of major visible blemishes, flaws, or damage. About $5 \mathrm{~kg}$ of muscadines were harvested for each genotype. The fruit was harvested into $0.9 \mathrm{~kg}$ clamshells (2-lbs) (about six clamshells per genotype), placed in an ice chest chilled with ice packs, and transported to the University of Arkansas Department of Food Science in Fayetteville, AR. Grapes were randomized by moving the fruit from the harvest clamshells and placing the fruit into new $0.9-\mathrm{kg}$ clamshells. The physiochemical and descriptive sensory attributes of the muscadines were evaluated in this study.

\section{Physiochemical analysis}

Fruit for physiochemical analysis was analyzed in triplicate per genotype. Each replication was a clamshell. The physiochemical analysis included physical and compositional analyses of the muscadines evaluated at harvest. For each genotype and replication, five randomly selected berries were used for analysis of both the physical attributes (15 berries) and composition attributes ( 15 berries).

Physical. Each whole berry was weighed and the firmness was measured immediately after harvest. Then, the seeds of each berry were removed, weighed, and counted. Berry weight and total seed weight were measured on a digital scale (PA224 Analytic Balance; Ohaus Corporation, Parsippany, NJ). Individual seed weight was calculated (total seed weight/number of seeds). Firmness of the berries was measured using a Stable Micro Systems TA.XT.plus texture analyzer (Texture Technologies Corporation, Hamilton, MA). The berries were placed on the texture unit vertically, stem scar down. Using the $2-\mathrm{mm}$ diameter probe, at a rate of $2 \mathrm{~mm} / \mathrm{s}$ with a trigger force of $0.02 \mathrm{~N}$, the flesh firmness and skin firmness were measured. Skin firmness, the force required to puncture the skin of the berry divided by the distance traveled before the berry skin was ruptured, was measured in $\mathrm{N} \cdot \mathrm{mm}^{-1}$. Flesh firmness was measured consecutively as the probe entered the flesh and was measured as force in $\mathrm{N}$.

Composition. The juice from the berries was measured for composition (soluble solids, $\mathrm{pH}$, and titratable acidity). The whole berries were frozen $\left(-10{ }^{\circ} \mathrm{C}\right)$, thawed, and squeezed to extract the juice for analysis. The $\mathrm{pH}$ and titratable acidity were measured using the Titrino plus 862 compact titrosampler (Metrohm AG, Herisan, Switzerland) with the electrode standardized to $\mathrm{pH} 4.00$, 7.00 , and 10.00 buffers. Titratable acidity was determined using $\approx 6 \mathrm{~g}$ of juice diluted with $50 \mathrm{~mL}$ deionized, degassed water with titration using $0.1 \mathrm{~N}$ sodium hydroxide to an end point of $\mathrm{pH}$ 8.2. Titratable acidity was expressed as percentage of tartaric acid. Soluble solids (expressed as percent) were measured using an Abbe Mark II refractometer (Bausch and Lomb; Scientific Instrument, Keene, NH). The ratio of soluble solids to titratable acidity was calculated.
Organic acids and sugars of the juice were determined using high-performance liquid chromatography (HPLC). The juice was filtered through a $0.45-\mu \mathrm{m}$ nylon filter (VWR International, Radnor, PA) and analyzed using HPLC. Glucose, fructose, tartaric acid, isocitric acid, and malic acid of the juice were measured using previously established HPLC procedures (Segantini et al., 2018; Walker et al., 2003). The HPLC was equipped with a Bio-Rad HPLC Organic Acid Analysis Aminex HPX-87H ion exclusion column $(300 \times 7.8 \mathrm{~mm})$, a Bio-Rad HPLC fast acid analysis column $(100 \times 7.8 \mathrm{~mm})$, and a BioRad HPLC column for fermentation monitoring $(150 \times 7.8 \mathrm{~mm})$ in series $($ Bio-Rad, Hercules, CA). A Bio-Rad Micro-Guard Cation-H refill cartridge $(30 \times 4.5 \mathrm{~mm})$ was used for a guard column. The columns were maintained at $65^{\circ} \mathrm{C}$ by a temperature control unit. The mobile phase consisted of a $\mathrm{pH} 2.28$ solution of sulfuric acid and water with a resistivity of $18 \mathrm{M}$, obtained from a Millipore Milli-Q reagent water system, with a $45 \mathrm{~mL} /$ min flow rate. The solvent delivery system was a Waters 515 HPLC pump equipped with a Waters 717 plus autosampler (Waters Corporation, Milford, MA). Injection volumes were $10 \mu \mathrm{L}$ for all samples and run time for completion was $35 \mathrm{~min}$. A Waters 410 differential refractometer to measure refractive index connected in series with a Waters 996 photodiode array detector monitored the eluting compounds. Tartaric, isocitric, and malic acids were detected by photodiode array at $210 \mathrm{~nm}$, and glucose and fructose were detected by the differential refractometer. Peaks were quantified using external standard calibration based on peak height estimation with baseline integration. Individual sugars and acids, total sugars (glucose + fructose), and total organic acids (tartaric + isocitric + malic) were expressed as $\mathrm{g} / 100 \mathrm{~g}$.

\section{Descriptive sensory analysis}

Descriptive sensory analysis was performed at the Sensory and Consumer Research Center at the University of Arkansas, Fayetteville, AR. After harvest, the fruit was stored overnight at $2{ }^{\circ} \mathrm{C}$ at $85 \%$ to $89 \%$ relative humidity for sensory. The fruit was removed from cold storage, gently rinsed, and placed on trays to air-dry. Each panelist evaluated fruit samples (five berries per sample) for each genotype in duplicate. The fruit was served monadically (one at a time) at room temperature $\left(25^{\circ} \mathrm{C}\right)$ on plates labeled with three-digit codes in a randomized complete block design. Serving order was randomized across each replication to prevent presentation order bias. Panelists were instructed to cleanse their palates with unsalted crackers and water between samples. Expectorant cups were provided. Panelists were trained to use a modified Sensory Spectrum method (Meilgaard et al., 2007), an objective method for describing the intensity of attributes in products using references for the attributes. Intensities of the aroma and aromatics were based on the universal scale, where a saltine was equal to a 3.0, applesauce was equal to a 7.0, orange juice was equal to a 10.0, grape juice was equal to a 14.0, and Big Red Gum ${ }^{\circledR}$ (Mars, Inc., MeLean, VA) was equal to a 15.0. The eight panelists developed a lexicon of descriptive sensory terms through consensus during orientation and practice sessions for the attributes of muscadine grapes (Table 1). The same descriptive panel evaluated the muscadine grapes for attributes including aroma $(n=9)$, external appearance $(n=7)$, internal appearance $(n=3)$, basic tastes $(n=3)$, aromatics $(n=10)$, feeling factors $(n=2)$, and texture $(n=7)$. These attributes were evaluated using a 15 -point scale, where $0=$ less of an attribute and $15=$ more of an attribute, except the number of seeds which were counted, and stem scar $(0=$ no scar; $1=$ scar present).

\section{Design and statistical analysis}

After harvest, the fruit from each of the six genotypes were completely randomized and placed into new clamshells. Statistical analyses were conducted using $\mathrm{JMP}^{\circledR}$ (version 13.2.0; SAS Institute, Cary, NC). Physiochemical attributes were evaluated in triplicate and descriptive sensory attributes were evaluated in duplicate. A univariate analysis of variance was used to determine the significance of the main factor (genotype). Tukey's honestly significant difference test was used to detect significant differences $(P<0.05)$ among means at the $95 \%$ significance level for physiochemical attributes. Least significant difference test was used to detect significant differences $(P<0.05)$ among means for sensory data. Pairwise correlations using multivariate analysis were used to verify the relationship between/ within attributes at a $P$ value of 0.05 .

\section{Results and Discussion}

Physiochemical attributes. The six muscadine genotypes were evaluated for physiochemical attributes (physical and composition), and physiochemical attributes varied significantly for most attributes. AM-74 (14.38 g) had the highest berry weight and seed weight $(0.12 \mathrm{~g})$ (Table 2). 'Summit' $(9.25 \mathrm{~g})$ had the lowest berry weight. Threlfall et al. (2007) found similar berry weight for 'Summit', but slightly lower weights for 'Ison' and 'Nesbitt' grapes also grown in Arkansas. AM-83 $(0.09 \mathrm{~g})$ had the lowest seed weight. Berry size may have been affected by sex of the plant as 'Summit' and AM-74 were female vines, whereas the other four genotypes were perfect-flowered vines. Although Williams (1957) found that female vines produce larger berries, the female vines in this study produced both large and small berries. There were no significant differences in seed number between these genotypes, but berries had about three seeds (ranging from one to four).

AM-83 had the highest skin firmness $\left(1.48 \mathrm{~N} \cdot \mathrm{mm}^{-1}\right)$ and flesh firmness $(2.14 \mathrm{~N})$. AM-9 $\left(0.85 \mathrm{~N} \cdot \mathrm{mm}^{-1}\right)$ had the lowest skin firmness and 'Nesbitt' $(0.89 \mathrm{~N})$ had the lowest flesh firmness (Table 2). Barchenger 


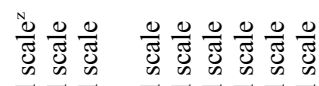

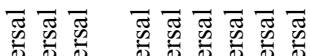

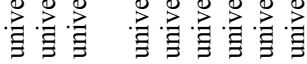

ธี ธี ธี ธี ฮี ธี

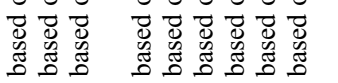

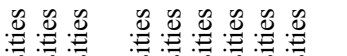

IIIIII

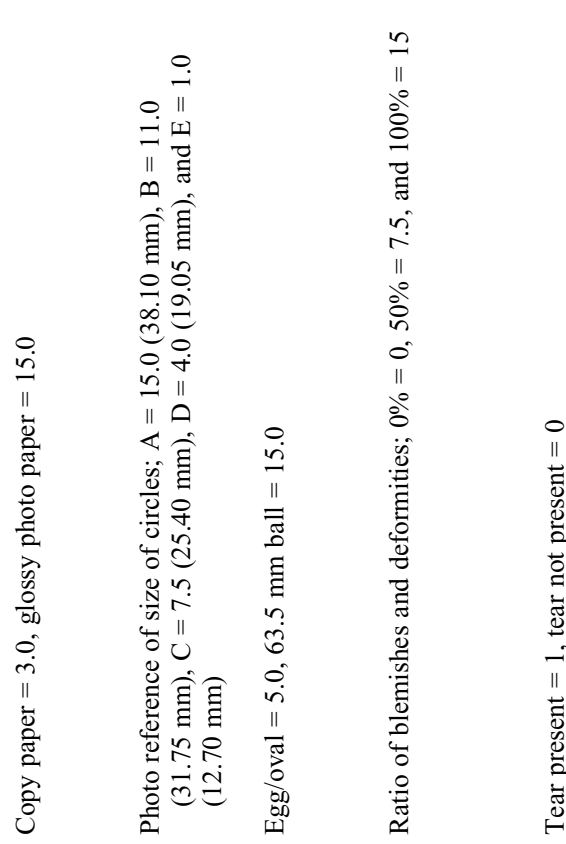

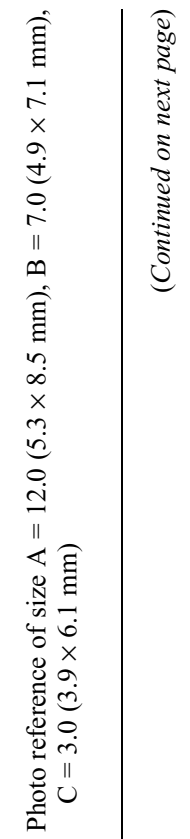

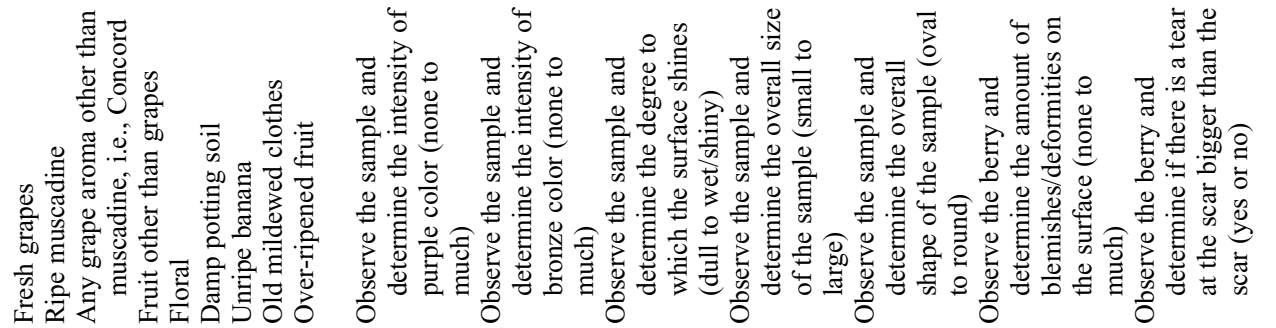
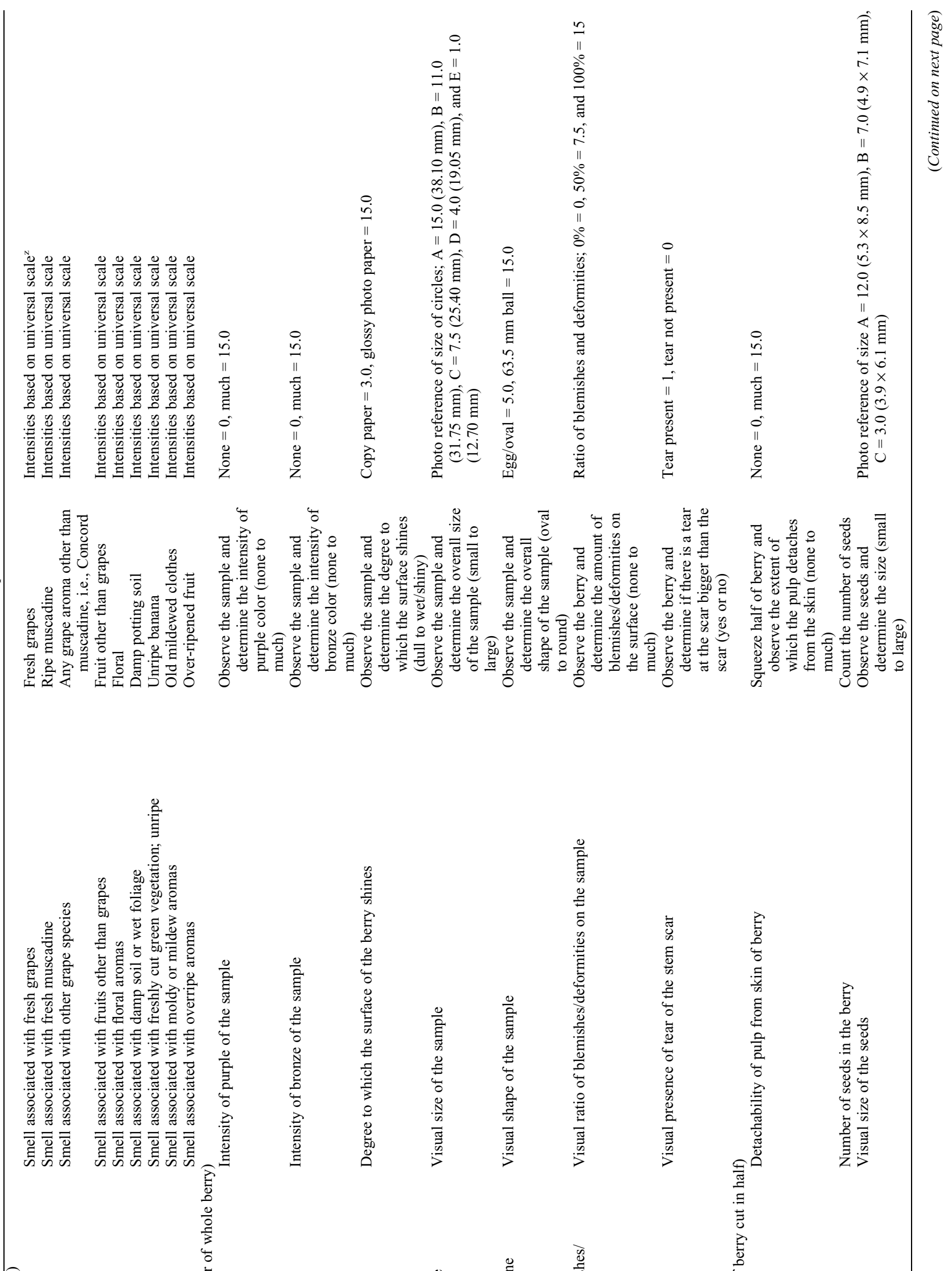

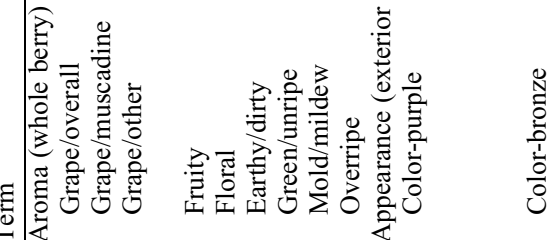
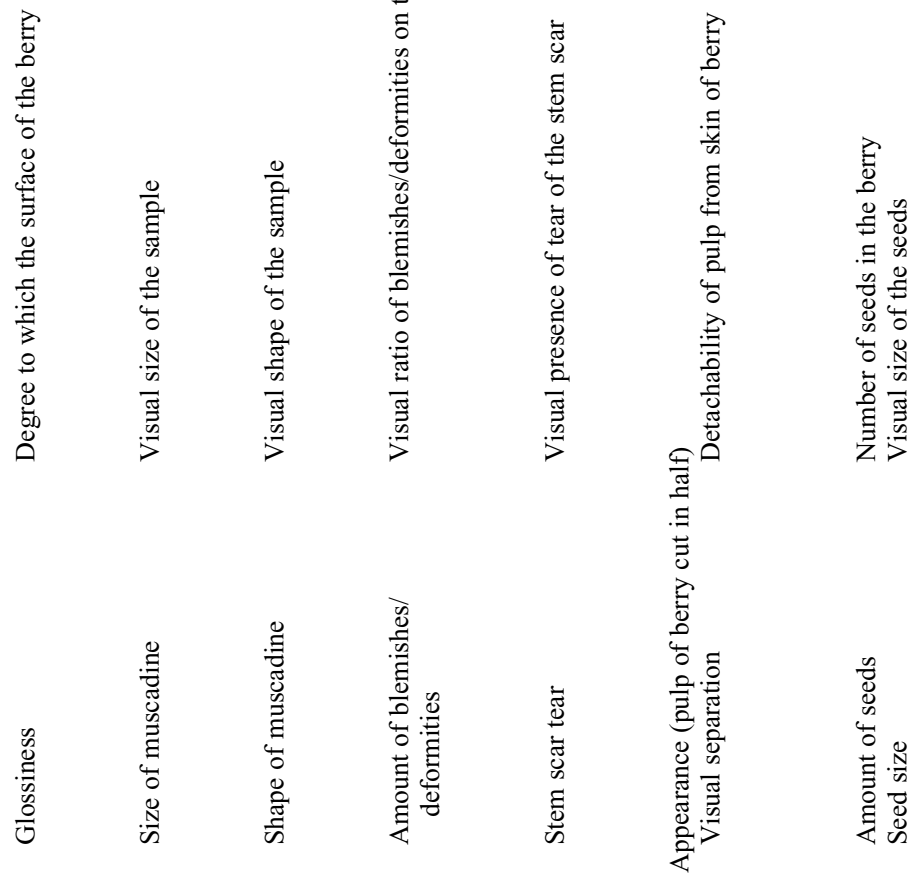


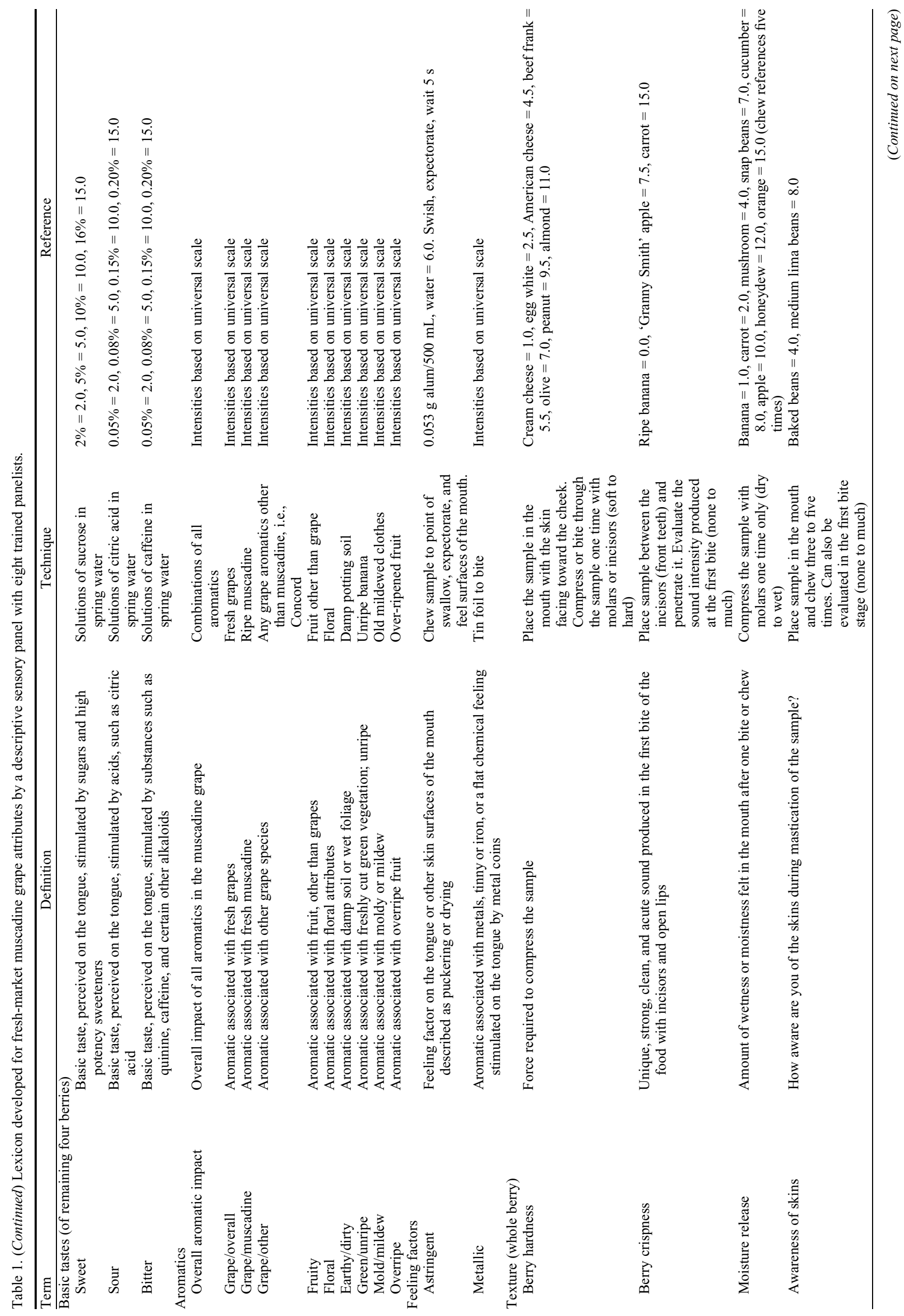

HortScience Vol. 53(11) November 2018 
et al. (2015b) found that the force required to penetrate the muscadine skin ranged from 6 to $13 \mathrm{~N}$ at harvest, similar to findings in this study of 5.31-7.50 N (data not shown). Conner (2013) reported flesh firmness ranging from 0.65 to $3.06 \mathrm{~N}$ in a study of 26 muscadine grape genotypes grown in Georgia. In that study, they also determined that flesh force or firmness was one of the most useful characteristics for screening in a breeding program to determine Vitis vinifera-like characteristics. Vitis vinifera grapes typically had higher flesh firmness and lower skin firmness than muscadine grapes. Therefore, Conner (2013) suggested selecting for increased flesh firmness and lower skin firmness in muscadine breeding programs. In this study, AM-83 was the most $V$. vinifera-like with respect to flesh firmness, but AM-9 was the most $V$. vinifera-like with respect to skin firmness.

'Summit' had the highest soluble solids $(15.40 \%)$ and lowest titratable acidity $(0.54 \%)$. 'Nesbitt' had the lowest soluble solids $(12.73 \%)$ and 'Ison' had the highest titratable acidity (1.01\%) (Table 2). AM-83 (3.33) had the highest $\mathrm{pH}$ and 'Ison' (2.88) had the lowest. Threlfall et al. (2007) found similar soluble solids, $\mathrm{pH}$, and titratable acidity levels for eight muscadine genotypes grown in Arkansas. The ratio of soluble solids to titratable acidity has proven useful for understanding how consumers perceived the balance of sugar to acid in fruit such as peaches and nectarines (Crisosto and Crisosto, 2005). 'Summit' (28.49) had the highest soluble solids/titratable acidity ratio and 'Ison' (13.12) the lowest (Fig. 1). Walker et al. (2001) indicated preferred soluble solids/titratable acidity ratios of 24-33 for muscadine grapes. Flora (1979) and Threlfall et al. (2007) also found similar preferred ratios for muscadine juice. Using these established parameters, three of the six genotypes (AM-9, AM-74, and 'Summit') in this study had an ideal ratio, two had almost ideal ratios (AM-83 and 'Nesbitt'), and one less than ideal ('Ison'). 'Ison' had a higher titratable acidity than the other genotypes. Although the soluble solids were similar, the greater titratable acidity led to the lower ratio observed.

Total organic acids and total sugars (Table 2) and individual organic acids and sugars (data not shown) were not significantly affected by the genotype. Overall, total sugars ranged from 6.17 to $9.75 \mathrm{~g} / 100 \mathrm{~g}$, and the total organic acids ranged from 0.50 to $0.84 \mathrm{~g} / 100 \mathrm{~g}$. Glucose and fructose were present in the fruit in an $\approx 1: 1$ ratio, with an average glucose content of $4.14 \mathrm{~g} / 100 \mathrm{~g}$ and fructose content of $3.81 \mathrm{~g} / 100 \mathrm{~g}$ (data not shown). Tartaric acid was the predominant acid in the muscadines with an average tartaric acid content of $0.37 \mathrm{~g} / 100 \mathrm{~g}$, isocitric acid content of $0.11 \mathrm{~g} / 100 \mathrm{~g}$, and malic acid content of $0.21 \mathrm{~g} / 100 \mathrm{~g}$ (data not shown). Similar organic acid and sugar contents have been observed in Arkansas-grown muscadines (Striegler et al., 2005). 
Table 2. Physical attributes for fresh-market muscadine genotypes, Clarksville, AR (2017).

\begin{tabular}{|c|c|c|c|c|c|c|c|c|c|c|}
\hline Genotype & $\begin{array}{l}\text { Berry skin } \\
\text { color }\end{array}$ & $\begin{array}{l}\text { Berry } \\
\text { wt (g) }\end{array}$ & $\begin{array}{l}\text { Seed } \\
\text { wt (g) }\end{array}$ & $\begin{array}{l}\text { Skin firmness } \\
\left(\mathrm{N} \cdot \mathrm{mm}^{-1}\right)\end{array}$ & $\begin{array}{c}\text { Flesh } \\
\text { firmness }(\mathrm{N})\end{array}$ & $\begin{array}{l}\text { Soluble } \\
\text { solids (\%) }\end{array}$ & $\mathrm{pH}$ & $\begin{array}{c}\text { Titratable } \\
\text { acidity }(\%)^{z}\end{array}$ & $\begin{array}{c}\text { Total } \\
\text { sugars }(\mathrm{g} / 100 \mathrm{~g})\end{array}$ & $\begin{array}{l}\text { Total organic } \\
\text { acids }(\mathrm{g} / 100 \mathrm{~g})\end{array}$ \\
\hline AM-74 & Bronze & $14.38 \mathrm{a}$ & $0.12 \mathrm{a}$ & $1.36 \mathrm{ab}$ & $1.13 \mathrm{ab}$ & $13.63 \mathrm{bc}$ & $3.08 \mathrm{bc}$ & $0.57 \mathrm{c}$ & 9.75 & 0.63 \\
\hline AM-83 & Black & $9.92 \mathrm{~b}$ & $0.09 \mathrm{~d}$ & $1.48 \mathrm{a}$ & $2.14 \mathrm{a}$ & $13.27 \mathrm{bc}$ & $3.33 \mathrm{a}$ & $0.64 \mathrm{bc}$ & 6.17 & 0.65 \\
\hline Nesbitt & Black & $10.10 \mathrm{~b}$ & $0.12 a b c$ & $1.40 \mathrm{ab}$ & $0.89 \mathrm{~b}$ & $12.73 \mathrm{c}$ & $3.03 \mathrm{~cd}$ & $0.76 \mathrm{~b}$ & 8.16 & 0.84 \\
\hline Summit & Bronze & $9.25 \mathrm{~b}$ & $0.10 \mathrm{~cd}$ & $1.18 \mathrm{ab}$ & $1.72 \mathrm{ab}$ & $15.40 \mathrm{a}$ & $3.19 \mathrm{ab}$ & $0.54 \mathrm{c}$ & 9.72 & 0.74 \\
\hline$P$ value & & $<0.0001$ & 0.0003 & 0.0073 & 0.0149 & 0.0002 & $<0.0001$ & $<0.0001$ & 0.9276 & 0.9309 \\
\hline
\end{tabular}

${ }^{\mathrm{z}}$ Titratable acidity expressed as $\%$ tartaric acid.

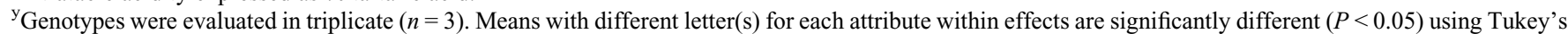
honestly significant difference test.

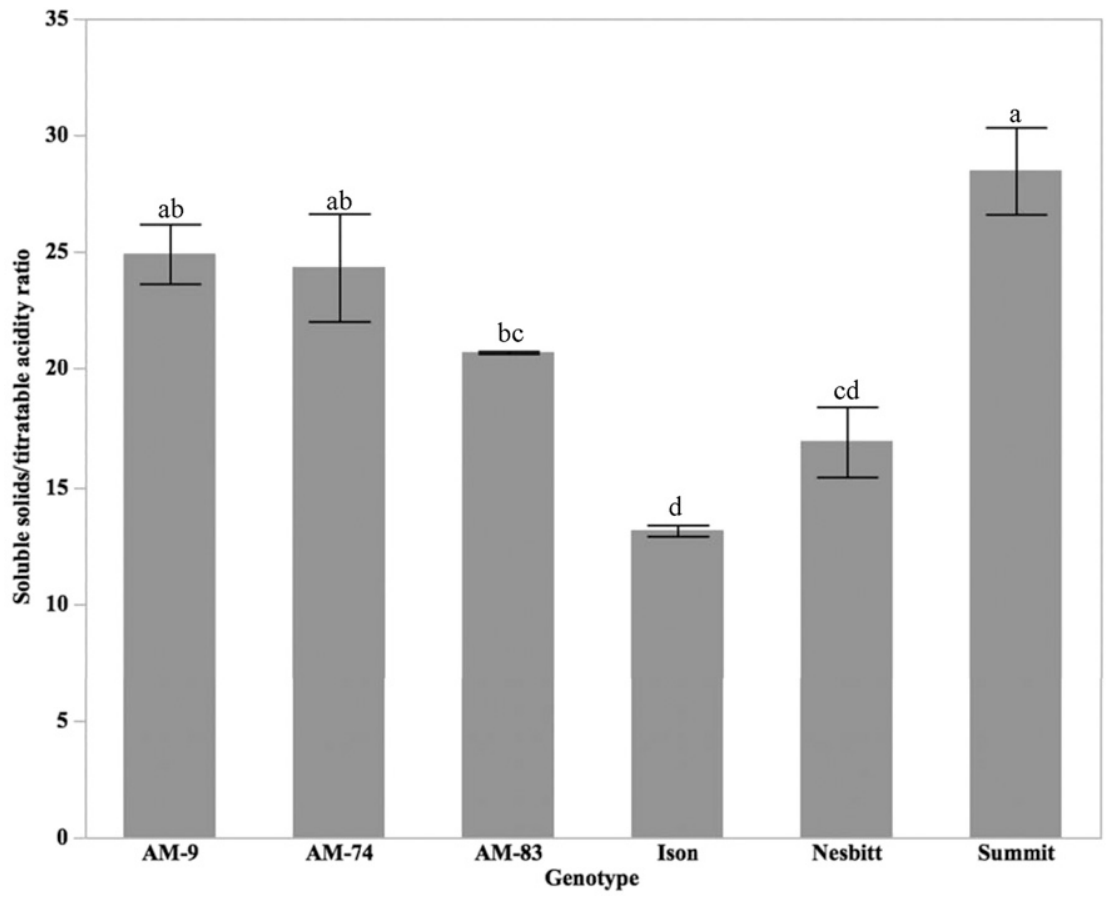

Fig. 1. Soluble solids/titratable acidity ratio of fresh-market muscadine genotypes, Clarksville, AR (2017) Each standard error bar is constructed using $1 \mathrm{se}$ from the mean. Genotypes were evaluated in triplicate $(n=3)$. Means with different letter(s) are significantly different $(P<0.05)$ using Tukey's honestly significant difference test.

Sensory attributes. Sensory analysis has been shown to explain cultivar characteristics better than instrumental analysis measurements alone. A descriptive sensory lexicon for Arkansas-grown muscadine grapes was created and used to evaluate the six genotypes (Table 1). The panelists evaluated the fruit on seven categories of attributes that included aroma, external appearance, internal appearance, aromatics, basic tastes, feeling factors, and texture. Within each of the seven categories, multiple attributes were evaluated. The results are reported in the order that the panelists evaluated the seven categories.

The panelists evaluated aroma (grape/ overall, grape/muscadine, grape/other, fruity, floral, earthy/dirty, mold/mildew, green/unripe, and overripe) of five whole, intact berries (Table 3). All of the aroma attributes were less than 6.5 on the 15-point scale, indicating lowmid aroma intensity. Of the aroma attributes, the panelists detected differences between genotypes in grape/overall, grape/muscadine, and fruity. The panelists did not detect differences in grape/other, floral, earthy/dirty, green/ unripe, or mold/mildew, which were low values $(\leq 0.8)$, and did not detect any overripe aromas. AM-74 had the highest grape/overall and grape/muscadine aroma and AM- 83 had the least. AM-9 had the greatest fruity aroma, and AM-83 had none.

Of the seven external appearance attributes, the panelists detected differences among the genotypes for all attributes except the amount of blemishes/deformities (Table 3). The panelists found that the muscadines had a low amount of blemishes/ deformities (3.9). AM-83 had the most color-purple, glossiness, and oblong shape. AM-74 had the most color-bronze and largest size. 'Summit' had the least glossiness (most dull exterior) and smallest size along with 'Nesbitt'. AM-9 had the roundest shape. In terms of stem scar, AM-9 had no visible stems scars, and AM-74 and 'Nesbitt' had more visible stem scars than the other genotypes (data not shown).
After the panelists evaluated external appearance, the muscadine berry was sliced in half from the stem scar down, and internal appearance (visual separation, number of seeds, and seed size) was evaluated (Table 3). Seed size (6.9) was not different among the genotypes, with seed size closest to the reference $\mathrm{B}=4.9 \times 7.1 \mathrm{~mm}$. The descriptive number of seeds ranged from 2.6 to 3.6 and was similar to physicochemical results (average of three seeds). Visual separation was the detachability of pulp from the skin of the berry. AM- 83 had the least visual separation and most seeds. 'Ison' had the most visual separation, and AM-74 had the fewest seeds. In a consumer study by Degner and Mathis (1980), the primary reason consumers did not purchase muscadines in Florida was the presence of seeds. Current muscadine breeding efforts are aimed at maintaining traditional muscadine traits such as aroma, flavor, and texture, while obtaining the complete absence of seeds.

Basic tastes (sweet, sour, and bitter) were evaluated (Table 4). Panelists detected differences among the genotypes for sweetness and sourness but not bitterness (1.0). Sweetness ranged from 6.3 to 7.9 , with a score of five which was equal to a $5 \%$ solution of sucrose. Sourness ranged from 2.7 to 3.9, with a reference of two which was equal to a $0.05 \%$ solution of citric acid. AM-74 was the most sweet and AM-83 was the least. 'Ison' was the sourest and 'Summit' was the least.

Panelists then evaluated the aromatics (olfactory perception caused by volatile substances released from a product in the mouth; retronasal) of the muscadines (Table 4). All of the aromatic attributes were less than 8.5 on the 15-point scale, indicating mid-low aromatic intensity. Of the attributes, the panelists detected differences between genotypes in overall aromatic impact, grape/ overall, and overripe, but all other attributes were not significantly different. AM- 83 had the least overall aromatic impact and grape/ overall aromatics. 'Summit' had the most overall aromatic impact; however, AM-74 had the most grape/overall aromatics. Although the overripe attribute was significant, these values were mostly zero (data not shown). There were no differences in grape/ muscadine (7.5) aromatics for these genotypes. The panelists found that the fruit had low levels of grape/other (0.4), fruity (0.9), 
Table 3. Descriptive sensory aroma and external and internal appearance attributes of muscadine genotypes evaluated on a 15 -point scale $(0=$ less of the attribute; $15=$ more of the attribute in terms of intensity), Clarksville, AR (2017).

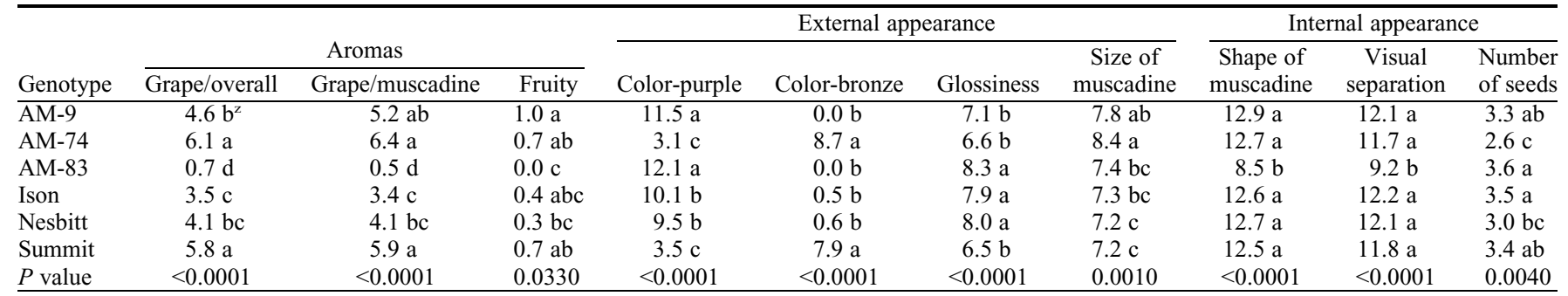

${ }^{\mathrm{z}}$ Genotypes were evaluated in duplicate by eight trained panelists. Means with different letter(s) for each attribute are significantly different $(P<0.05)$ using least significant difference.

Table 4. Descriptive sensory basic tastes, aromatics, and feeling factor attributes of muscadine genotypes evaluated on a 15 -point scale $(0=$ less of the attribute; $15=$ more of the attribute in terms of intensity), Clarksville, AR (2017).

\begin{tabular}{|c|c|c|c|c|c|}
\hline \multirow[b]{2}{*}{ Genotype } & \multicolumn{2}{|c|}{ Basic tastes } & \multicolumn{2}{|c|}{ Aromatics } & \multirow{2}{*}{$\frac{\text { Feeling factor }}{\text { Metallic }}$} \\
\hline & Sweet & Sour & Overall aromatic impact & Grape/overall & \\
\hline$\overline{\mathrm{AM}-9}$ & $7.4 \mathrm{abc}^{\mathrm{z}}$ & $3.2 \mathrm{bcd}$ & $8.1 \mathrm{abc}$ & $6.7 \mathrm{ab}$ & $1.4 \mathrm{abc}$ \\
\hline AM-74 & $7.9 \mathrm{a}$ & $2.9 \mathrm{~cd}$ & $8.3 \mathrm{ab}$ & $6.9 \mathrm{a}$ & $1.2 \mathrm{~d}$ \\
\hline AM-83 & $6.3 \mathrm{~d}$ & $3.3 \mathrm{bc}$ & $7.1 \mathrm{~d}$ & $5.9 \mathrm{~d}$ & $1.4 \mathrm{bc}$ \\
\hline Ison & $6.7 \mathrm{~cd}$ & $3.9 \mathrm{a}$ & $7.7 \mathrm{c}$ & $6.2 \mathrm{~cd}$ & $1.6 \mathrm{a}$ \\
\hline Nesbitt & $7.0 \mathrm{bcd}$ & $3.7 \mathrm{ab}$ & $7.9 \mathrm{bc}$ & $6.4 \mathrm{bc}$ & $1.5 \mathrm{ab}$ \\
\hline Summit & $7.6 \mathrm{ab}$ & $2.7 \mathrm{~d}$ & $8.4 \mathrm{a}$ & $6.8 \mathrm{ab}$ & $1.3 \mathrm{~cd}$ \\
\hline$P$ value & 0.0020 & 0.0010 & $<0.0001$ & $<0.0001$ & 0.0010 \\
\hline
\end{tabular}

${ }^{\mathrm{z}}$ Genotypes were evaluated in duplicate by eight trained panelists. Means with different letter(s) for each attribute are significantly different $(P<0.05)$ using least significant difference.

floral (0.7), earthy/dirty (1.0), green/unripe (1.8), and mold/mildew (0.3) aromatics.

Feeling factors (astringent and metallic) were evaluated (Table 4). Panelists detected differences among the genotypes for metallic but not astringent (6.7). The reference for metallic is tin foil to bite. Metallic feeling factor was low (1.2-1.6). AM-74 was the least metallic, and 'Ison' was the most metallic.

The panelists evaluated texture (berry hardness, berry crispness, moisture release, awareness of skins, detachability, fibrousness between teeth, and seed separation) for four berries in the five-berry sample. However, no differences were found among the genotypes for these attributes. All genotypes had a midhigh intensity with respect to awareness of skins (13.0), detachability (11.9), and seed separation (10.3). Panelists found a medium intensity for all genotypes with respect to berry hardness (8.3) and moisture release (9.9). Finally, these genotypes had low to mid intensity for berry crispness (3.6) and fibrousness (4.1). Because the panelists were unable to detect differences in texture of muscadine grapes, possibly reestablishing the texture standards within a narrower range could lead to more discrimination between the texture attributes or having the panelists evaluate a shorter lexicon with texture only.

Correlations between physiochemical and sensory attributes. Physical attributes with more than one correlation to sensory attributes were presented in Table 5 and in text, whereas attributes with one correlation are only discussed in the text. Of the physical attributes, berry weight was positively correlated with the size of the muscadine $(r=$ $0.95)$, floral aromatics $(r=0.86)$, and moisture release $(r=0.83)$, and negatively correlated with the descriptive number of seeds $(r=-0.84)$. Seed weight was positively correlated with green/unripe aromas $(r=$ $0.87)$. Seed number was correlated with stem scar tear $(r=-0.82)$, descriptive number of seeds $(r=0.98)$, floral aromatics $(r=-0.94)$, and mold/mildew aromatics $(r=0.92)$.

Of the texture attributes, flesh firmness was negatively correlated with the shape of the muscadine $(r=-0.82)$ and visual separation $(r=-0.82)$, and positively correlated with grape/muscadine aromatics $(r=0.83)$. The firmer the berry, the more oval and less detachability of pulp from the skin of berry. Skin firmness was positively correlated with the amount of blemishes/deformities $(r=$ 0.97). Therefore, higher skin firmness indicated a less visually desirable berry in this study. Blemishes/deformities evaluated by the panel were attributed to naturally occurring dark spots or healed sections of the fruit. These blemishes/deformities were superficially present, and in certain instances, they had a different texture than the skin of the berry. This texture difference may have led to the correlation observed with skin firmness.

Of the composition attributes, soluble solids were negatively correlated with grape/other aromatics $(r=-0.87)$. The $\mathrm{pH}$ was negatively correlated with green/unripe aromas $(r=-0.86)$ and positively correlated with berry crispness $(r=0.83)$. Therefore, increased $\mathrm{pH}$ indicated less green/unripe aroma and crisper berries. Titratable acidity was negatively correlated with grape/other aromas $(r=-0.83)$ and positively correlated with sourness $(r=0.90)$, green/unripe aromatics $(r=0.83)$, and metallic feeling factor $(r=0.87)$. Interestingly, titratable acidity was the predominant factor of sour taste in the grapes as indicated by the lack of correlation of $\mathrm{pH}$ with sourness. More than soluble solids, $\mathrm{pH}$, or titratable acidity, the soluble solids/titratable acidity ratio had the most correlations to descriptive attributes. The soluble solids/titratable acidity ratio was correlated with five sensory attributes, including a positive correlation with grape/ other aromas $(r=0.86)$ and negative correlations with sour $(r=-0.97)$, grape/other aromatics $(r=-0.89)$, green/unripe aromatics $(r=-0.86)$, and metallic feeling factor $(r=$ $-0.85)$. Composition of the berries, especially the relationships observed between composition and aromas/aromatics, may have been attributed to the overall ripeness of the muscadine berries. A study by Walker et al. (2001) found that sensory panelists were best able to identify ripeness of muscadine grapes by the soluble solids to titratable acidity ratio. As the soluble solids and $\mathrm{pH}$ increased, and the titratable acidity decreased, the panelists perceived the fruit as more ripe. Therefore, the positive correlation with aromatic attributes and soluble solids, and the negative correlation with aroma attributes and titratable acidity may indicate that the riper the berries are, the greater the aroma/aromatic impact will be.

Correlations were found with respect to the total sugars, glucose, and fructose with descriptive sensory attributes. Total sugars were correlated with 12 sensory attributes, including positive correlations to grape/overall aroma $(r=0.86)$, grape/muscadine aroma $(r=0.82)$, floral aroma $(r=0.87)$, colorbronze $(r=0.92)$, sweet $(r=0.84)$, overall aromatic impact $(r=0.84)$, grape/overall aromatics $(r=0.81)$, fruity aromatics $(r=$ $0.94)$, and negative correlations to colorpurple $(r=-0.97)$, bitter $(r=-0.86)$, mold/ mildew aromatics $(r=-0.88)$, and astringent feeling factor $(r=-0.87)$. Data for glucose and fructose correlation are not shown in Table 5 but listed in the text. Glucose and fructose were both positively correlated ( $r=$ $0.85-0.94)$ with grape/overall aroma, floral aroma, color-bronze, overall aromatic impact, and fruity aromatics, and negatively correlated $(r=-0.85-0.97)$ with colorpurple, bitter, mold/mildew aromatics, and astringent feeling factor. Fructose was positively correlated ( $r=0.82-0.87)$ with grape/ muscadine aroma, and sweet, grape/overall aromatics, and negatively correlated with glossiness $(r=-0.83)$. From these results, total and individual sugars were clearly important in increasing the presence of desirable aromas and aromatics, such as floral 


\begin{tabular}{|c|c|c|c|c|c|c|c|}
\hline Sensory attributes & Berry wt (g) & Seed (no.) & Flesh firmness $(\mathrm{N})$ & $\mathrm{pH}$ & $\begin{array}{c}\text { Titratable } \\
\text { acidity }(\%)\end{array}$ & $\begin{array}{c}\text { Soluble solids/titratable } \\
\text { acidity ratio }\end{array}$ & $\begin{array}{c}\text { Total sugars } \\
(\mathrm{g} / 100 \mathrm{~g})\end{array}$ \\
\hline \multicolumn{8}{|l|}{ Aromas } \\
\hline Grape/overall & $0.44^{z}$ & -0.67 & -0.58 & -0.28 & -0.33 & 0.49 & $0.86 *$ \\
\hline Grape/muscadine & 0.46 & -0.66 & -0.60 & -0.23 & -0.37 & 0.52 & $0.82 *$ \\
\hline Grape/other & -0.13 & -0.23 & 0.26 & 0.59 & $-0.83 *$ & $0.86 *$ & 0.57 \\
\hline Floral & 0.27 & -0.63 & -0.50 & -0.13 & -0.45 & 0.59 & $0.87 *$ \\
\hline Green/unripe & 0.27 & -0.58 & -0.67 & $-0.86 *$ & 0.36 & -0.23 & 0.67 \\
\hline \multicolumn{8}{|l|}{ External appearance } \\
\hline Color-purple & -0.47 & 0.70 & 0.14 & 0.18 & 0.40 & -0.54 & -0.97 \\
\hline Color-bronze & 0.51 & -0.67 & -0.02 & -0.04 & -0.51 & 0.63 & $0.92 *$ \\
\hline Size of muscadine & $0.95 *$ & -0.63 & -0.29 & 0.10 & -0.42 & 0.35 & 0.30 \\
\hline Shape of muscadine & 0.24 & -0.45 & $-0.82 *$ & -0.57 & 0.10 & 0.06 & 0.54 \\
\hline Stem scar tear & 0.62 & $-\mathbf{0 . 8 2 *}$ & -0.57 & -0.58 & 0.17 & -0.30 & 0.51 \\
\hline \multicolumn{8}{|l|}{ Internal appearance } \\
\hline Visual separation & 0.12 & -0.33 & $-0.82 *$ & -0.63 & 0.24 & -0.06 & 0.44 \\
\hline Number of seeds & $-0.84 *$ & $0.98 *$ & 0.69 & 0.26 & 0.28 & -0.18 & -0.66 \\
\hline \multicolumn{8}{|l|}{ Basic tastes } \\
\hline Sweet & 0.58 & -0.73 & -0.47 & -0.04 & -0.56 & 0.67 & $0.84 *$ \\
\hline Sour & -0.30 & 0.36 & -0.29 & -0.58 & $0.90 *$ & $-0.97 *$ & -0.62 \\
\hline Bitter & -0.33 & 0.62 & -0.06 & 0.21 & 0.25 & -0.33 & $-0.86 *$ \\
\hline \multicolumn{8}{|l|}{ Aromatics } \\
\hline Overall aromatic impact & 0.34 & -0.60 & -0.53 & -0.18 & -0.40 & 0.57 & $0.84 *$ \\
\hline Grape/overall & 0.50 & -0.67 & -0.49 & -0.03 & -0.55 & 0.67 & $0.81 *$ \\
\hline Grape/muscadine & -0.14 & 0.38 & $0.83 *$ & 0.76 & -0.35 & 0.20 & -0.46 \\
\hline Grape/other & -0.22 & 0.36 & -0.21 & -0.32 & 0.73 & $-0.89 *$ & -0.77 \\
\hline Fruity & 0.41 & -0.69 & -0.02 & -0.11 & -0.43 & 0.52 & $0.94 *$ \\
\hline Floral & $0.86^{*}$ & $-0.94^{*}$ & -0.50 & -0.41 & -0.13 & 0.08 & 0.72 \\
\hline Green/unripe & -0.43 & 0.22 & -0.41 & -0.66 & $0.83 *$ & $-0.86 *$ & -0.35 \\
\hline Mold/mildew & -0.69 & $0.92 *$ & 0.44 & 0.42 & 0.18 & -0.20 & $-\mathbf{0 . 8 8 *}$ \\
\hline \multicolumn{8}{|l|}{ Texture } \\
\hline Moisture release & 0.83* & -0.81 & -0.49 & -0.43 & -0.14 & 0.23 & 0.76 \\
\hline Berry crispness & -0.19 & 0.35 & 0.51 & $0.83 *$ & -0.40 & 0.16 & -0.59 \\
\hline \multicolumn{8}{|l|}{ Feeling factors } \\
\hline Astringent & -0.30 & 0.58 & -0.15 & -0.23 & 0.69 & -0.76 & $-0.87 *$ \\
\hline Metallic & -0.60 & 0.61 & -0.12 & -0.48 & $0.87^{*}$ & $-\mathbf{0 . 8 5 *}$ & -0.66 \\
\hline
\end{tabular}

${ }^{\mathrm{z}}$ Bold values with asterisks $(*)$ have significant correlations $(P<0.05)$ using a multivariate pairwise analysis.

and fruity, as seen by the positive correlations with overall aromatic impact.

Total organic acids were not correlated with any of the descriptive sensory attributes. Of the individual organic acids evaluated, isocitric acid was negatively correlated with the shape of the muscadine $(r=-0.82)$, tartaric acid was negatively correlated with overripe aromatics $(r=-0.82)$, and malic acid was positively correlated $(r=0.83-0.85)$ with sour and green/unripe aromatics. Although not the predominant acid, malic acid was the only acid to show correlation with sour taste, indicating that malic acid may be of greater importance than isocitric or tartaric acids, with the perception of sourness in the muscadine grapes. In addition, the presence of tartaric acid had a negative effect on the aromatic attribute of overripe.

\section{Conclusion}

The descriptive sensory panelists differentiated between genotypes for external appearance, internal appearance, and basic taste attributes, more specifically with positive attributes rather than negative, but poorly with the selected aroma, aromatic, and texture attributes. This indicated that of the attributes evaluated in this study, descriptive sensory analysis was best suited for appearance and basic taste attributes. Conversely, of the texture attributes evaluated by the panelists, no differentiation was seen, indicating that better descriptors and references need to be selected for further studies. The intent of this study was to establish the descriptive sensory lexicon for future muscadine evaluations. Descriptive sensory analysis of muscadine grape breeding lines is not always possible because of limited amount of fruit, so establishing correlations between descriptive sensory attributes and physiochemical attributes could be useful for muscadine breeding. Physiochemical attributes such as total sugars and soluble solids/titratable acidity ratio had the most significant correlations with descriptive sensory attributes. For soluble solids/titratable acidity ratio, a higher ratio indicated that the fruit was perceived as riper and potentially more desirable as negative attributes such as green/unripe aromatics, sour basic taste, and metallic feeling factor decreased. Therefore, at harvest, fruit with higher soluble solids/titratable acidity ratios should be selected. In addition, higher total sugar content indicated a positive effect on the pleasant aromatics of the fruit and a negative effect of the displeasing aromatics and external appearance. Retention of muscadine aroma and aromatics, while improving fruit texture, is an important goal for muscadine breeding programs. Although the panelists were unable to distinguish between genotypes for texture attributes, analytical texture analysis was correlated with external and internal appearance and aromatic attributes, indicating that firmness (skin and flesh) plays a role in how the berry is perceived both visually and aromatically. A descriptive sensory lexicon for fresh-market muscadine grapes was created. Descriptive sensory and physiochemical analysis has the potential to identify important attributes of fresh-market muscadine grapes. Evaluating descriptive sensory, consumer sensory, and physicochemical attributes could provide data about what attributes consumers like and dislike about muscadines.

\section{Literature Cited}

Barchenger, D.W., J.R. Clark, R.T. Threlfall, L.R. Howard, and C.R. Brownmiller. 2015a. Evaluation of physiochemical and storability attributes of muscadine grapes (Vitis rotundifolia Michx.). HortScience 50:104-111.

Barchenger, D.W., J.R. Clark, R.T. Threlfall, L.R. Howard, and C.R. Brownmiller. 2015b. Nutraceutical changes in muscadine grape and grape segments during storage. J. Amer. Pomol. Soc. 69:66-73.

Bouquet, A. 1981. Resistance to grape fanleaf virus in muscadine grape inoculated with Xiphinema index. Plant Dis. 65:791-793.

Brown, K., C. Sims, A. Odabasi, L. Bartoshuk, P. Conner, and D. Gray. 2016. Consumer acceptability of fresh-market muscadine grapes. J. Food Sci. 81:S2808-S2816.

Carpio, C.E., C.D. Safley, and E.B. Poling. 2008. Estimated costs and investment analysis of producing and harvesting muscadine grapes in the southeastern United States. HortTechnology 18:308-317. 
Cline, B. and C. Fisk. 2006. Overview of muscadine grape acreage, cultivars and production areas in the southeastern U.S. Muscadine Grape Workshop for Cooperative Extension Agents, 2006. The Southern Region Small Fruit Consortium. 25 July 2007. <http://www. smallfruits.org/CoAgentTraining/Sept06Training/ No1Muscadine_acres_and_cultivars.pdf $>$.

Conner, P.J. 2013. Instrumental textural analysis of muscadine grape germplasm. HortScience 48:1130-1134.

Contador, L., M. Díaz, E. Hernández, P. Shinya, and R. Infante. 2017. The relationship between instrumental tests and sensory determinations of peach and nectarine texture. Eur. J. Hort. Sci. 81:189-196.

Crisosto, C.H. and G.M. Crisosto. 2005. Relationship between ripe soluble solids concentration (RSSC) and consumer acceptance of high and low acid melting flesh peach and nectarine (Prunus persica (L.) Batsch) cultivars. Postharvest Biol. Technol. 38:239246.

Degner, R.L. and K. Mathis. 1980. Consumer acceptance of muscadine grapes. Proc. Annu. Meet. Fla. State Hort. Soc. 93:140-143.
Flora, L.F. 1979. Optimum quality parameters of muscadine grape juices, beverages, and blends. J. Food Qual. 2:219-229.

Meilgaard, M.C., B.T. Carr, and G.V. Civille. 2007. Sensory evaluation techniques. 4th ed. CRC Press, Boca Raton, FL.

Meullenet, J.F., C. Lovely, R. Threlfall, J.R. Morris, and R.K. Striegler. 2008. An ideal point density plot method for determining an optimal sensory profile for muscadine grape juice. Food Qual. Prefer. 19:210-219.

Olien, W.C. 1990. The muscadine grape: Botany, viticulture, history, and current industry. HortScience 25:732-739.

Ren, Z. and J. Lu. 2002. Muscadine rootstock increased the resistance of Florida hybrid bunch grape cv. Blanc du Bois to Pierce and Anthracnose diseases. Proc. Annu. Meet. Fla. State Hort. Soc. 115:108-110.

Segantini, D.M., R.T. Threlfall, J.R. Clark, L.R. Howard, and C.R. Brownmiller. 2018. Physiochemical changes in floricane and primocane blackberries harvested from primocane genotypes. HortScience 53:9-15.

Striegler, R.K., J.R. Morris, P.M. Carter, J.R. Clark, R.T. Threlfall, and L.R. Howard. 2005. Yield, quality, and nutraceutical potential of selected muscadine cultivars grown in southwestern Arkansas. HortTechnology 15:276-284.

Threlfall, R.T., J.R. Morris, J.F. Meullenet, and R.K. Striegler. 2007. Sensory characteristics, composition, and nutraceutical content of juice from Vitis rotundifolia (muscadine) cultivars. Amer. J. Enol. Viticult. 58:268-273.

Trappey, A.F., C.E. Johnson, and P.W. Wilson. 2007. Consumer acceptance of mayhaw ( $\mathrm{Cra}$ taegus opaca Hook. and Arn.) juice blended with muscadine grape (Vitis rotundifolia Michx.) juice. Intl. J. Fruit Sci. 6:53-65.

Walker, T., J. Morris, R. Threlfall, and G. Main. 2003. Analysis of wine components in Cynthiana and Syrah wines. J. Agr. Food Chem. 51:1543-1547.

Walker, T.L., J.R. Morris, R.T. Threlfall, G.L. Main, O. Lamikanra, and S. Leong. 2001. Density separation, storage, shelf life, and sensory evaluation of 'Fry' muscadine grapes. HortScience 36:941-945.

Williams, C. 1957. Relation of berry size to flower type of seedlings in muscadine grape crosses. Proc. Amer. Soc. Hort. Sci. 69:254-260. 